\title{
Research on the Design of the Effective Transmission Scheduling Algorithm Based on the Factory Wireless Sensor Network
}

\author{
Yunyi Zhang ${ }^{1{ }^{1 *}}$, Shuru Liu ${ }^{1}$, Ran Zhang ${ }^{1}$ and Wei Wei ${ }^{2,3}$ \\ ${ }^{I}$ College of Computer and Communication Engineering, Zhengzhou University of Light Industry, Zhengzhou 450002, \\ China \\ ${ }^{2}$ School of Computer Science and Engineering, Xi'an University of Technology, Xi'an, 710048, China \\ ${ }^{3}$ Shaanxi Key Laboratory for Network Computing and Security Technology, China
}

\begin{abstract}
In order to make the sensing data in the monitoring equipment's sensor nodes reach to the monitoring center as soon as possible and deal with the equipment which has some problems, the network time delay should be guaranteed in the accessible Time Delay Bound. The paper mainly researches the transmission scheduling algorithm with the chain framework, which adopts the Token to do the transmission in the chain and then arranges the transmission order in each node for avoiding the occurrence of the collision and offering the guarantee of the time delay. The node order in each chain is the order of data packets which are transmitted by nodes, while the route of the data packet transmission is according to the shortest route in the Sink Tree for reducing the time delay.
\end{abstract}

Keywords: Delay bound, scheduling algorithm, wireless sensor network.

\section{INTRODUCTION}

IEEE 802.11 adopts the medium storage method in the CSMA/CA (Carrier Sense Multiple Access/Collision Avoidance) and the DCF (Distributed Coordination Function) method to do the transmission in the wireless network. If the nodes in the mechanism of the CSMA/CA need to transmit the packets, the state of the medium must be monitored. If the medium is in the idle state, it can be transmitted. If the medium is in the busy sate, it must be delayed for some time. When the medium is in the free time, it can be transmitted after waiting for a Random Back off Time. The medium storage method can reduce the probability of the packet collision. With the increasing number of the nodes in the network, the probability of collision can be increased, the whole efficiency can be reduced and the transmission delay time of the packets can also be increased, obtained from $[1,2]$. If TDMA offers the protocol method based on the competition, the packet collision and delay caused by the nodes with the competition channel can be solved, and it has a higher Throughput and the bounded time delay. The advantage can make TDMA be applied in the application of Time-sensitive, such as the monitoring system of the automatic control and others [3]. Considering that the wireless sensor network can be applied in the monitoring of the factory equipment and the factory environment has the requirements of high timesensitive, the occurrence of the errors to the mechanical equipment can cause the reduction of the productivity so that the scheduling mechanism which does not need the precise synchronization requirement on the basis of the concept of TDMA, which is fit for the environment of the factory equipment monitoring [5-10]. The paper introduces the transmission scheduling protocol based on the chain, and the node order of the nodes is the order of the node's transmission data packet. The packet data include Token, and then the transmission among the nodes with the Token can reach to the synchronization. Later, if the number of the nodes in each chain is fixed, the bounded time delay of the packet transmission can be estimated in advance [11-18].

\section{RELATED RESEARCHES}

The CTSBD (Chain-based Transmission Scheduling with Bounded Delay) [4] builds the topology of the chain framework, as shown in the Fig. (1). If there are many Sink in the network, the upstream and downstream relationship among the Sink can be decided in advance. A sub chain between each Sink in the upstream and each Sink in the downstream can be set up, and the direction of the sub chain is the direction which is pointed by the Sink in the upstream to the Sink in the downstream. The display order of each node in the sub chain is from the Sink in the upstream to the Sink in the downstream, that is, the order of the Token transmission. When a certain node receives the Token from the upstream node, the node can have the power to transmit all ordered data packet completely. Later, the Token can be released to the downstream node. Before each node checks the transmitted data packet, the information of the route table should be checked. The data packet should transmit it to the nearest 


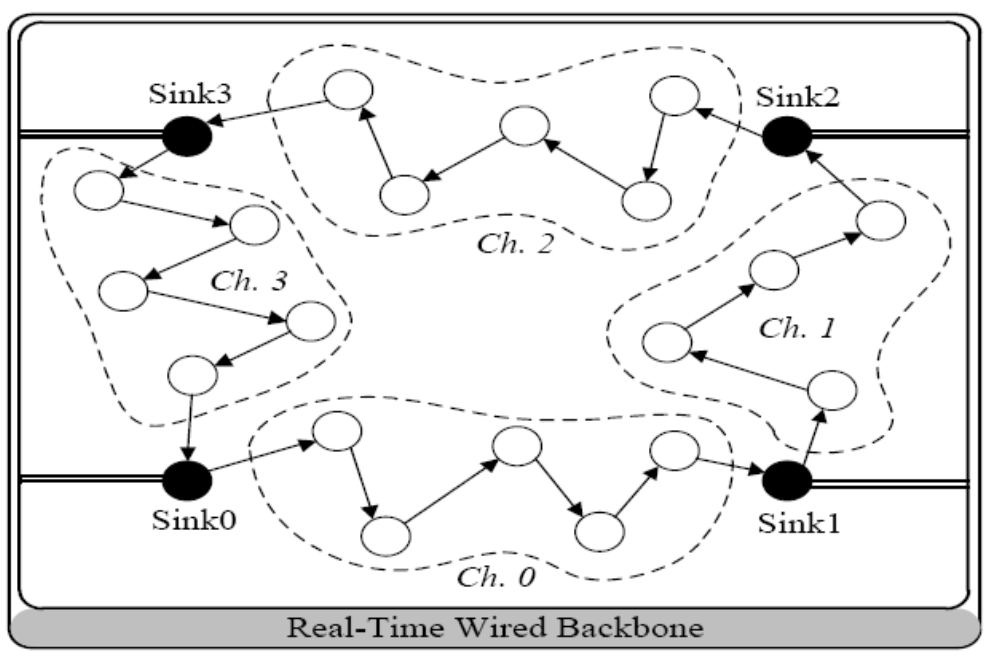

Fig. (1). Operational method of the CTSBD network.

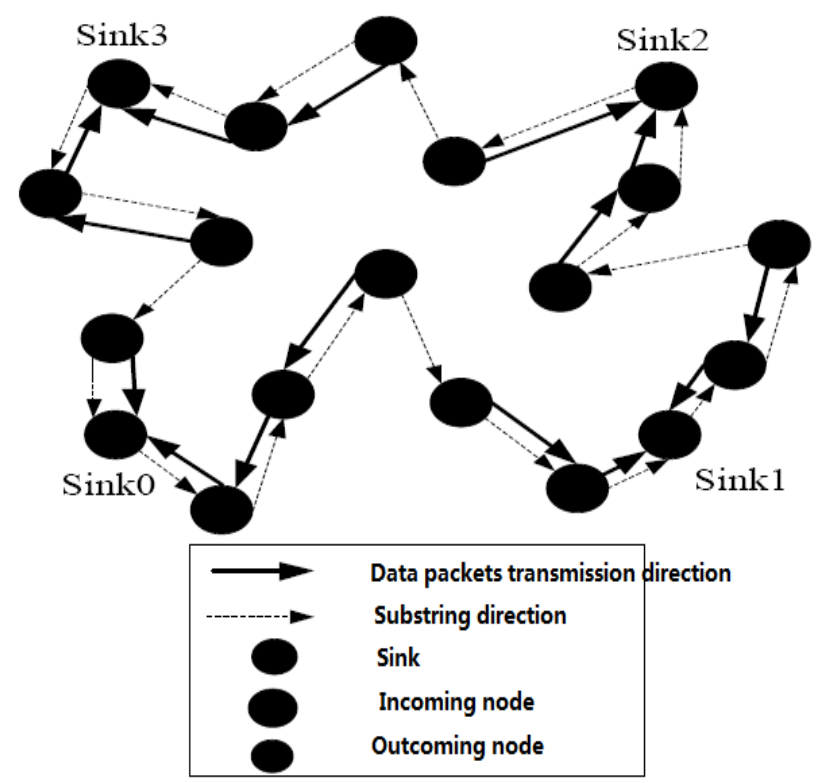

Fig. (2). Direction of the sub chain and the data packet transmitting direction of the nodes.

Sink away with itself so that the transmitting direction of the data packet can be opposite to the direction of the Token, as shown in the Fig. (2).

The paper researches the Robust Chain-based Transmission Scheduling with Bounded Delay, RCTSBD on the basis of the chain, which will modify the CTSBD [4]. The topology of the chain framework proposed in the previous CTSBD [4] has some disadvantages so that the upstream Sink and downstream Sink can not built the initial route and the transmission of the sensor data cannot be conducted. Therefore, the searching of the initial route and the building of the sub chain can be modified. In this way, a sub chain can be set up between each Sink and its downstream Sink. In addition, the direction of the sub chain is the direction by the upstream Sink points to the downstream Sink. After the sub chain forms, some nodes can be added and these nodes are called as the isolated nodes.

The data packet cannot be transmitted into the Sink for the isolated nodes cannot be added in the sub chain. The condition of producing the isolated nodes and the method of eliminating the isolated nodes should be discussed.

\section{RESEARCH ISSUE-THE SCHEDULING OF THE DATA PACKET TRANSMISSION}

\subsection{Network Framework}

RCTSBD adopts the network framework which is the same as the CTSBD [4], as shown in the Fig. (1). The sensor 
nodes are randomly displayed in everywhere according to the requirements and communicated with the method of the wireless (including Sink). The Sink node is built in many fixed positions in the factory and collects the data of the sensor nodes. Later, the data can be transmitted in the central monitoring center by the back-end wired network. The procedures of the transmitting the data packet includes the following steps: establishing the neighboring lists, establishing the route table, grouping the nodes and establishing the sub chain. All nodes (including Sink) adopt the same channel do to the transmission. The upstream Sink is communicated with the downstream Sink through the wired network. When the downstream Sink receives the Token, the wired network should notice the upstream Sink to send the next Token. Therefore, each Sink should be equipped with two WLAN network cards, and each operation is in the different channels, while other nodes just need to be equipped with one WLAN network card.

\subsection{The Establishment of the Route Table}

The route table is built by pack Sink broadcasts the HOP_TREE packet, and the HOP_TREE packet includes the address of the source node Sink, the Next Hop near to the source node Sink and the information of some hops. If the number of hops in the Sink is set up as 0 and the node receives the HOP_TREE packet, the number of hops is added one in the packet and the received number is the total hops of the node from the specific HOP TREE packet. Later, the computed hops, the transmission node of the packet and the Sink address should be recorded in the route table, and the HOP_TREE packet should be broadcast to the neighboring node.

When each node in the network builds the information of each Sink (including the Sink address, the number of the hop and next hop), the route table and its neighboring node list will notice the nearest Sink so that the route from each node to the nearest Sink can be confirmed. The route should be adopted to the application of the transmission data packet.

\subsection{The group of the Node}

Before building the sub chain among the Sink, each Sink will divide the Offspring of the Sink Tree into two groups: the Incoming group and the Outgoing group. The Incoming group means that the Offspring node has a smaller distance between the Upstream Sink and the Downstream Sink. Otherwise, it is called as the Outgoing group. In order to reduce the system cost and improve the establishing flexibility, the nodes in the network are unnecessary to know its geographic position so that the grouping algorithm adopts the hops to replace the comparison of the geographic position.

If the hops are used as the distance, the hops from the nodes to the Upstream Sink are the same as the hops from the nodes to the Downstream Sink so that these nodes can not be grouped exactly. Therefore, the paper introduces a judging method to solve the problem. The neighbors in the node should be judged and these neighboring nodes can be divided into the number proportion of the the Incoming group and the Outgoing group. If the number of the Incoming group is more than the number of the Outgoing group, the node enters the Incoming group. Otherwise, the node enters the Outgoing group.

\subsection{The Establishment of the Chain}

\subsubsection{The Searching of the Initial Route}

After the group is finished, each Sink will notice the node list belonging to the Incoming group to the Upstream Sink. Later, each Sink can have the node data in the Outgoing group and the node list in the Incoming group noticed by the Downstream Sink. These nodes belong to the members of the sub chain. The procedure is initiated by the Sink whose address is the minimum and the Initiator Sink of the sub chain can transmit the ANNOUNCE packet to the Terminator Sink in the Downstream.

Firstly, the Initiator Sink should judge whether it can has the nodes in the Incoming group. If the Initiator Sink has the nodes in the Incoming group, the node which has a hop and belongs to the Incoming group should be selected as the first node in the initial route to transmit the packet. If the Initiator Sink has not the nodes in the Incoming group, it should judge whether it has the node which has two hops and belongs to the Incoming group. If the Initiator Sink has the node which has two hops and belongs to the Incoming group, it should search for the neighboring node which has two hops and belongs to the Incoming group. The neighboring node must be the node in the Outgoing group and has a hop away from the Initiator Sink. If the node in the Outgoing group which is accorded with the condition is found out, it can be changed into the node in the Incoming group and be regarded as the first node in the initial route to transmit the packet. If the Initiator Sink cannot find out the node in the Outgoing group which is accorded with the condition, can just has the node in the Outgoing group, the node nearest to the Terminator Sink should be selected from the Outgoing group as the first transmission node and the group type of the node should be changed into the Incoming group. The node in the changed Incoming group should be regarded as the firs $\mathrm{t}$ node in the initial route to transmit the packet.

When the first transmission node receives the ANNOUNCE packet, the address should be recorded in the packet and the last transmitter's address of the packet should also be recorded. Later, the next transmitter forwarding to the Terminator Sink in the route table should be checked whether it belongs to the member of the Incoming group in the packet. If it is the member of the Incoming group, the packet can be transmitted. Otherwise, check whether it is the member of the Outgoing group in the packet. If it is the member of the Outgoing group, the list in the Incoming group should be searched and a neighboring node nearer to the Terminator Sink should be selected as the next transmitter of the packet. Otherwise, the next transmission node belongs to the node of the Outgoing group or the Incoming group in the Terminator Sink. When the route table heads for 


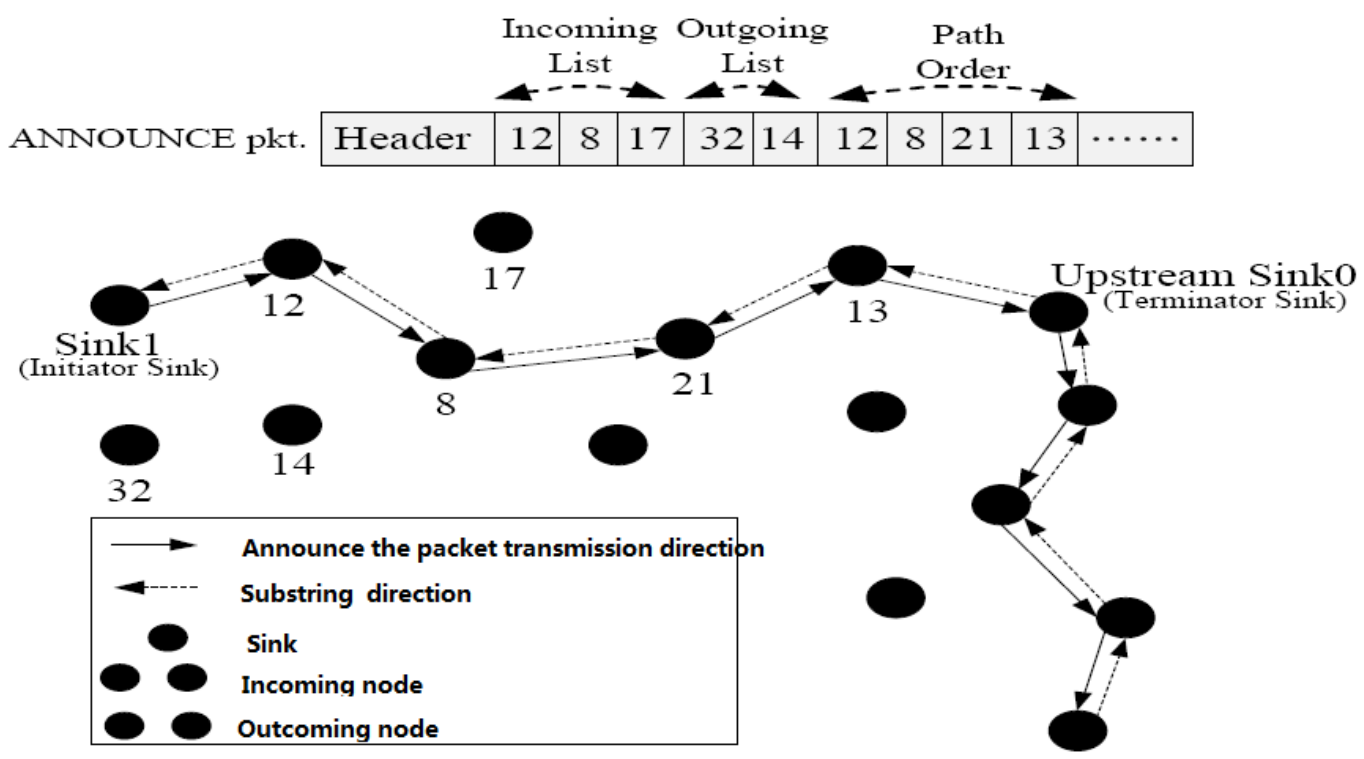

Fig. (3). Main route of the ANNOUNCE packet.

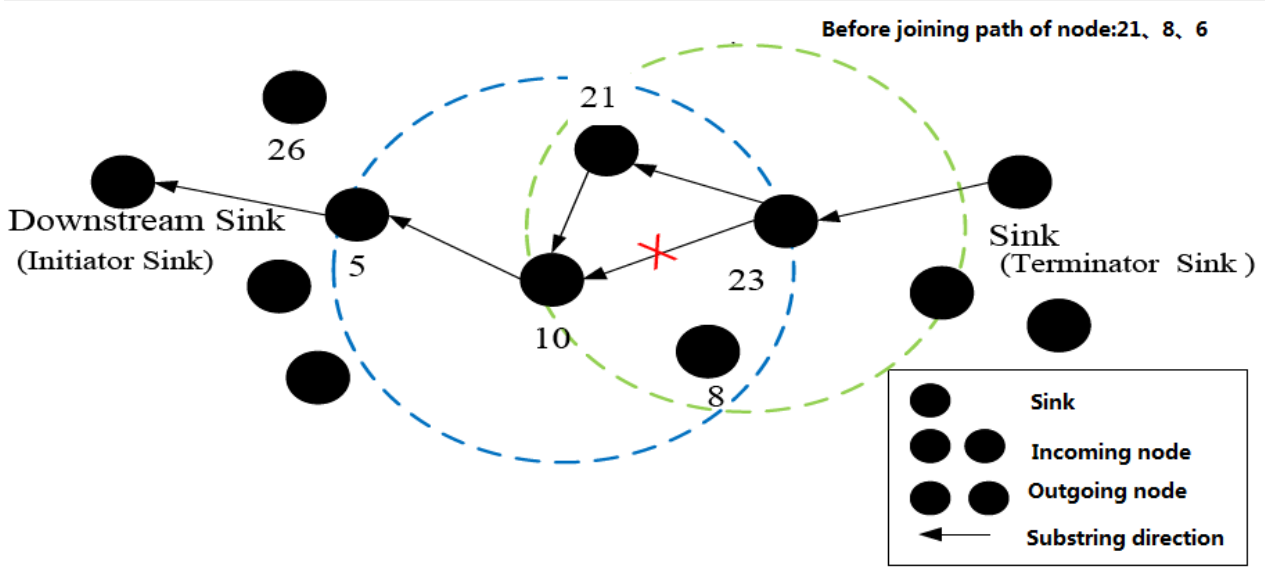

Fig. (4). The procedure of the adding route whose node is not added firstly.

the next step of the Terminator Sink and it cannot be accorded with the condition, the Outgoing group node in the next transmitter heading for the Terminator Sink should be changed into the Incoming group node. The repeated examination is to ensure that the nodes are the Incoming group member of the Initiator Sink after the packet is through the previous route of the initial route. The packet transmitter should record the address in the packet. After the last transmitter's address of the packet is recorded, the packet can be transmitted to the next node.

When the Terminator Sink receives the ANNOUNCE packet, the Incoming group node list in the packet should be recorded and then the recorded each node in the packet should be checked whether there are the members of the Incoming group. If there are the members of the Incoming group, the group type of the node should be changed into the Outgoing group and then another ANNOUNCE packet can be transmitted into the next Terminator Sink. The procedure should be operated repeatedly until the Sink whose address is the minimum receives the ANNOUNCE packet. In this way, the initial route among the Sink can be formed, and its operation method is as shown in the Fig. (3).

\subsection{The Establishment of the Sub Chain}

After the initial route between the Initiator Sink and Terminator Sink is established, each Sink will compare the Incoming group node list in the Downstream Sink adding the node in the initial route with the Outgoing group node list, and then the node list without adding into the initial route firstly should be summarized and the list should notice each node in the initial route. Each node in the route should begin to check whether the neighboring node can have the nodes without adding into the initial route. If there are the nodes, the nodes should be added. The procedure is explained with the use of the Fig. (4).

After the node 23 receives the node list (node 21, 8, 26) without adding into the initial route firstly, the neighboring 


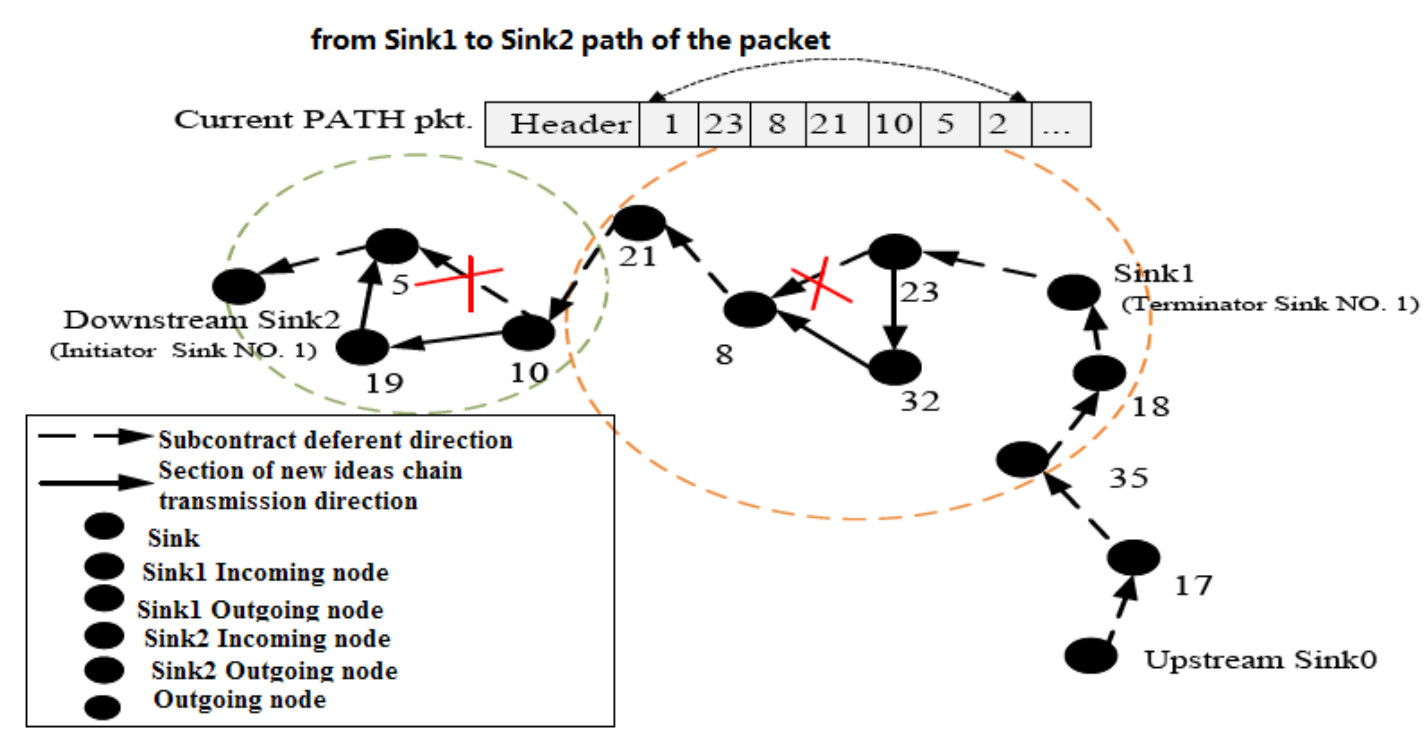

Fig. (5). Sink adds the nodes which have not added second times to the route of the sub chain.

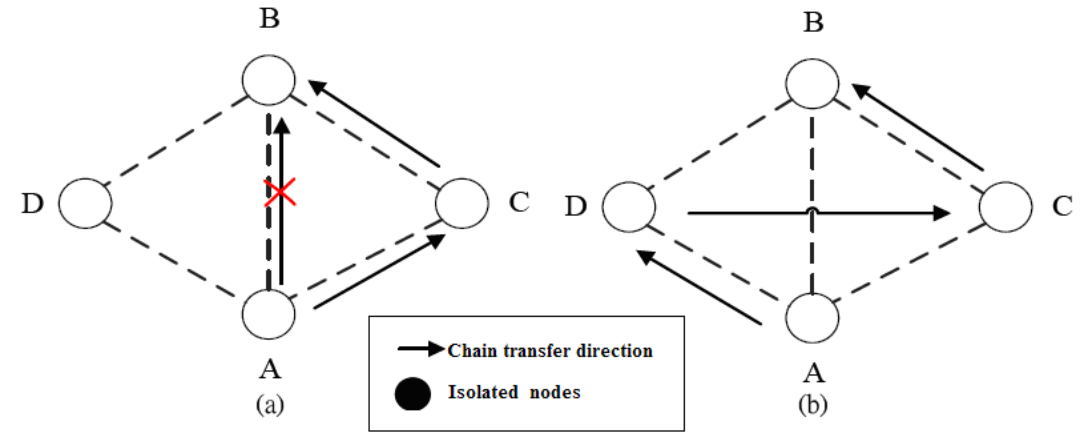

Fig. (6). The occurred situation and the solution of the isolated nodes in the network.

nodes should be checked, the neighboring node 21 and node 8 can be found out in the list. At first, the node 23 tries adding the node 21 into the route of the sub chain so that the node 23 can ask whether the node 10 and the node 21 in the Downstream is the neighbor of the node 10 . When the node 10 replies to the node 23 , the node 21 is the neighbor is the node 10 . The node 23 will change the next receiver transmitting the Token into the node 21 and the node 23 , later the node 23 should be noticed that its next Token receiver is the node 10 . Then, the node 23 tries adding the node 8 into the route again until the node 23 can be added into the route's node, and the node list which is added or not added should be transmitted into the Downstream node and the Downstream node searches for the node which is not added firstly.

When the node which is not added firstly to the network adds into the adding route, each Initiator Sink compares the route from the Offspring node to the Upstream Sink with the route from the Offspring node to the Downstream Sink. If the Offspring node (including the Incoming node and the Outgoing node) has the nodes which have not added, the nodes which have not added should be placed in the node list (including the Incoming node and the Outgoing node) which has not added second times. The Outgoing node list which has not added second times should notice the Upstream Sink (Terminator Sink) to do the node's second times adding, the procedure is explained with the use of the Fig. (5).

After using Terminator Sink NO. 1 (Sink 1) receives Initiator Sink No. 1 (Sink 2)'s Outgoing group node 19 and the Incoming group node 32, the node list which has not added second times should be summarized. The Current PATH should be transmitted into the Downstream node and do the second times node accession heading for the Downstream Sink 2.

After the sub chain establishes, these nodes which has not added are called as the isolated nodes. The Fig. (6a) is the example of happening the isolated node $\mathrm{D}$. The isolated node $\mathrm{D}$ can not becomes the neighbor with the node $\mathrm{C}$ in the present route so that the route cannot be added. When the isolated nodes happen, the communication range between the isolated D and other nodes near to the isolated D is increased. After the isolated $\mathrm{D}$ and the isolated $\mathrm{V}$ becomes the neighbor, it can be added in the route of the sub chain, as shown in the Fig. (6). 


\subsection{The Estimation of the Bounded Time Delay}

The estimated computing method of the Bounded time delay is as follows. If a data packet is produced within the total time $\mathrm{T}$ to each sensor node, the following formula (1) is the total time's computing method of each chain.

$$
\begin{gathered}
T=\sum_{j=1}^{|N m|} h_{j} \times T_{\text {one_p } p k t}=\sum_{j=1}^{|N m|} T_{j} \\
T_{\text {one_pkt }}=T_{\text {backoff }}+\text { DIFS }+T_{R T S}+T_{C T S}+T_{\text {DATA }} \\
+T_{A C K}+3 \times S I F S
\end{gathered}
$$

in which, Nm is the node set of the sub chain $\mathrm{m}$, hj is the hops whose node $\mathrm{j}$ in the $\mathrm{Nm}$ is away from the Sink $\mathrm{m}, \mathrm{Tj}$ is the needed time when the node $j$ transmits all packets in the buffer.

If the Leave node $\mathrm{i}$ in the Sink Tree transmits the information packet in the first round, the Token is handed in the next node and the data packets are produced, the packets must wait for the time unit in the node i's buffer. When it reaches to the second round, the node i can transmit the packet to the next transmission node $\mathrm{i}-1$. The route by the node $i$ transmits the data packets to the Sink is just the route by the node in the sub chain comes back to the Sink, and the maximum time delay will be produced. If the packet is in the node $i$, the scheduling delay, the queue delay and the transmitting delay is TS,i、TQ,I and TTXi respectively.

$T_{S, i}=T-T_{i}$

$T_{Q, i}=x_{i} \times T_{\text {one_pkt }}$

$T_{T X, i}=T_{\text {one_pkt }}$

in which, $x i$ is the number of the packets in which the node $i$ needs to be transmitted, that is, the number of the Downstream node in the node i. After the node $i$ is produced, the delay time by the packet reaches to the node $\mathrm{i}-1$ is $T_{L, i}=T_{s, i}+T_{Q, i}+T_{T, i, i}=T-T_{i}+\left(x_{i}+1\right) \times T_{\text {one }}$ plt $=T$.

In the same way, the middle node $\mathrm{k}$ is $k=1,2, \ldots, i-2$.

$T_{L, k}=T_{S, k}+T_{Q, k}+T_{T X, k}$

$=T-T_{k+1}$

The packet's total delay by the node i reaches to the Sink is as follows:

$$
\begin{aligned}
& T_{\max \text { Delay }}=\sum_{k=1}^{h_{i}} T_{L, k} \\
& =h_{i} \times T-T_{i}-\mathrm{L}-T_{3}-T_{2}
\end{aligned}
$$

Therefore, when the topology of the Sink Tree is confirmed, the maximum hi, xk and $\mathrm{Tk}(\mathrm{k}=\mathrm{i}, \mathrm{i}-1, \ldots, 1)$ can be known. Later, the Tmax Delay can be estimated.

\subsection{The Estimation of the Throughput}

The estimated computing method of the throughput is as follows. If each node produces a data packet which should be transmitted $100 \mathrm{~ms}$, the following formula (10) is the total cycle time's computing method of each chain. Each node adopts the 1 Mbps speed rate to transmit the data packet:

$$
\begin{aligned}
& T=N_{n} \times T_{k}+\sum_{i=1}^{|N n|} h_{i} \times\left[T_{\text {other }}+T_{\text {data }}\right] \\
& T_{\text {other }}=T_{\text {bacloff }}+D I F S+T_{R T S}+T_{C T S}+T_{A C K}+3 \times S I F S
\end{aligned}
$$

in which Tk is the transmitting time of the Token, TData is the transmitting time of each data packet, $\mathrm{Nn}$ is the node ste of the sub chain $n$, and $\mathrm{hj}$ is the hops whose node $\mathrm{j}$ in the $\mathrm{Nm}$ is away from the Sink. (Sink Tree Hop)

$$
\text { Throughput }=\frac{N_{n} \times T_{\text {Data }} \times 1 \mathrm{Mbps}}{\operatorname{Max}(T, 100 \mathrm{~ms})}
$$

\section{SIMULATION ENVIRONMENT AND RESULTS}

The environmental framework in the paper is to delay 4 Sinks in the $30 \mathrm{~m} \times 30 \mathrm{~m}$ environment, and the 4 Sinks positions are fixed, while the positions of other nodes are randomly delayed. If the wireless medium is the ideal channel, the Bit Error during the time of transmitting the packets should not be considered, the transmission packets is failed under the collided situation. If a data packet needs to be transmitted in each node $100 \mathrm{~ms}$, each node adopts the 1 Mbps speed rate to transmit the data packets. In this way, its size is 250 bytes and its simulated time is $60 \mathrm{~s}$.

The following Table $\mathbf{1}$ is the setting of the simulated parameters:

\subsection{The Influence of the Node Number in the Sub Chain to the Time Delay}

The situations of the different time delay in terms of the number of the node in the chain should be observed after the establishment of the sub chain, as shown in the Fig. (7). If there are many number of nodes in the sub chain, the number of the nodes which can have a higher time delay can also be increased so that the average time delay of the whole sub chain can be affected. If there is a little number of nodes in the sub chain, the number of the nodes which can have a higher time delay can also be reduced.

\subsection{The Influence of the Node Number in the Sub Chain to the Throughput}

The numbers of the nodes which are randomly delayed in the network are 35 (not including 4 Sinks) and then the sub chain is formed. The sub chain 0 has 10 nodes, the sub chain 
Table 1. The setting of the simulated parameters.

\begin{tabular}{|c|c|c|c|}
\hline Parameter & Value & Parameter & Salue time \\
\hline \hline Communication radius & $8 \mathrm{~m}, 9 \mathrm{~m}, 10 \mathrm{~m}, 11 \mathrm{~m}$ & CW min & 50 us \\
\hline PHY Header & 128 bits & SIFS & DIFS \\
\hline RTS & 288 bits + PHY Header & & 128 us \\
\hline CTS & 240 bits + PHY Header & \\
\hline ACK & 240 bits + PHY Header & \\
\hline
\end{tabular}

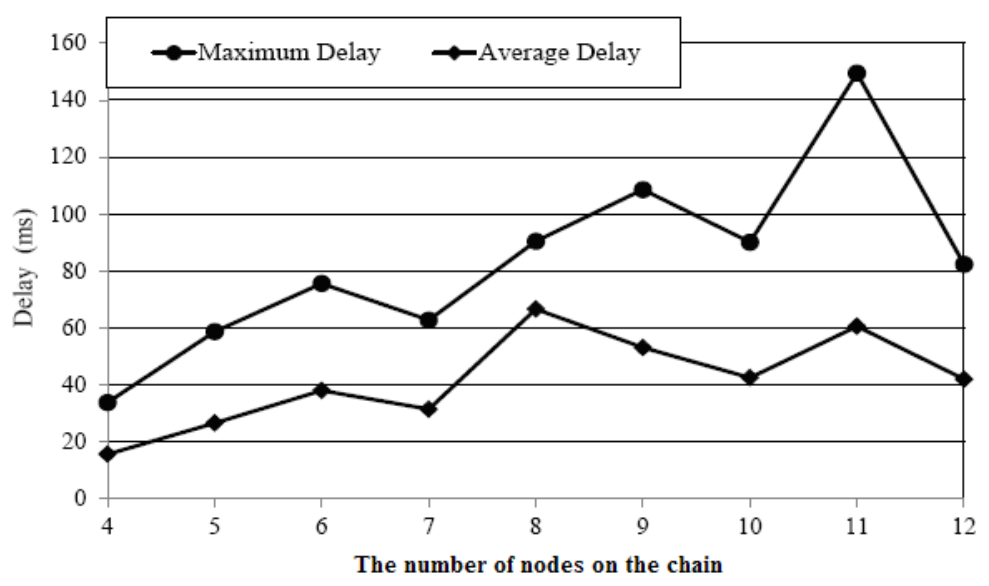

Fig. (7). The time delay of different node numbers in the sub chain.

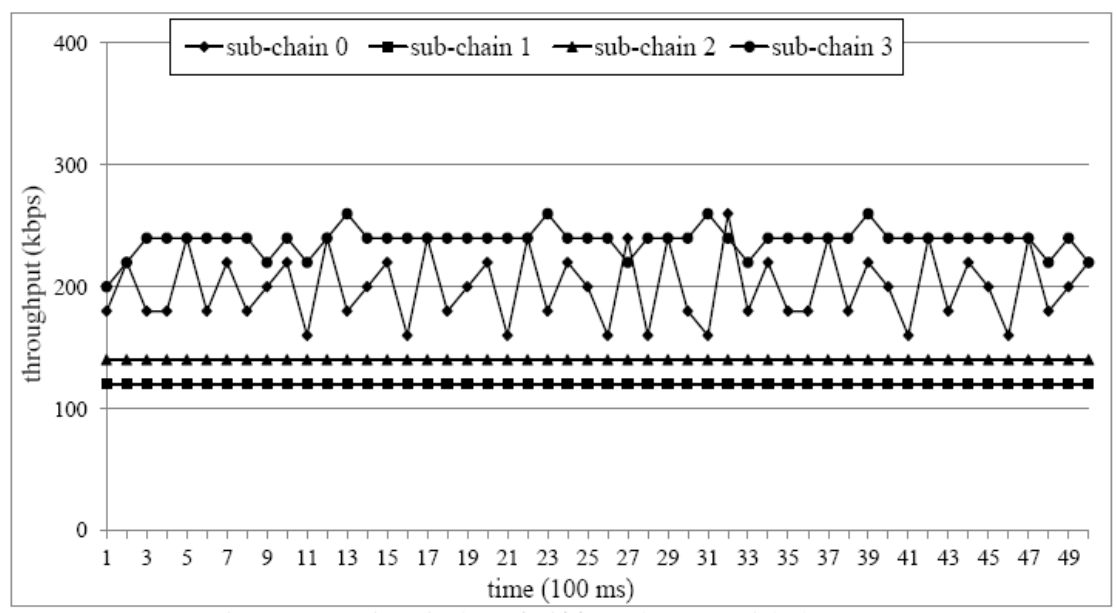

Fig. (8). The expression of the throughput of different node numbers in the network.

1 has 6 nodes and the sub chain 3 has 12 nodes. If each node produces 10 packets which need to be transmitted within $1 \mathrm{~s}$, the throughput of each sub chain each $100 \mathrm{~ms}$ should be observed and its observed times is 50 times. The throughput produced by the sub-chain 2 is $140 \mathrm{kbps}$ which is related to the node number of the sub chain, and the maximum throughput produced by the sub-chain 3 which is related to the node number of the sub chain and the nodes' average hops is $260 \mathrm{kbps}$, as shown in the Fig. (8).

\subsection{The Influence of the Node Number in the Sub Chain to the Transmission Rate}

The network formed by the CTSBD [4] can have the nodes which have not added into the sub chain (called as rage isolated nodes), as shown in the Fig. (9). The isolated nodes cannot transmit the data packets to the Sink and then the packets will be lost, while the RCTSBD make these isolated nodes add into the sun chain route so that each node in 


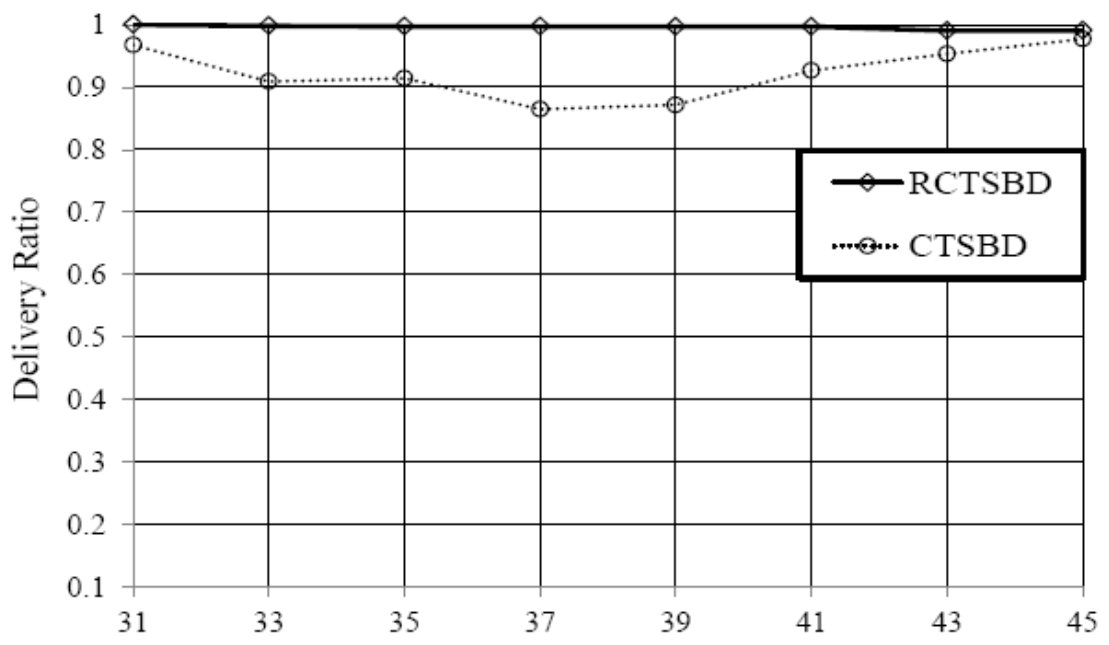

Fig. (9). The expression of the transmission rate of different node numbers in the network.

the network can have the chance to transmit the data packets to the Sink.

\section{SUMMARY}

Compared with the previous transmission scheduling in the CTSBD [4], the RCTSBD proposed in the paper has been improved. The positions of the nodes in the network are randomly distributed can easily make the distribution of the nodes no uniform, and then the isolated nodes will be existed after the route of the initial sub chain is established. The RCTSBD can add the isolated nodes into the route of the initial sub chain so that the problem of the isolated nodes can be solved. The number of the nodes in the sub chain can be increased, the throughput and the Delivery Ratio can also be improved because these isolated nodes add into the route of the sub chain.

\section{CONFLICT OF INTEREST}

The authors confirm that this article content has no conflicts of interest.

\section{ACKNOWLEDGEMENTS}

We would like to thank the anonymous reviewers for their valuable comments. We also sincerely appreciate Prof. Yong Qi for his valuable advices and helping. This program is supported by Scientific Research Program Funded by key scientific and technological project of Henan Province (No. 142102210081) and Shaanxi Provincial Education Department (Program No.2013JK1139) and Supported by China Postdoctoral Science Foundation (No.2013M542370) and supported by the Specialized Research Fund for the Doctoral Program of Higher Education of China (Grant No. 20136118120010). And this project is also supported by NSFC Grant (Program No. 11301414 and No.61272283 and No.61272283 and No.61472318).

\section{REFERENCES}

[1] S. Chu, and X. Wang, "Adaptive and distributed scheduling in heterogeneous MIMO-based ad hoc networks," In: Proceedings of IEEE MASS'09, Macau: IEEE, pp. 217-226, 2009.

[2] S. Chu, and X. Wang, "Opportunistic and cooperative spatial multiplexing in MIMO ad hoc networks," IEEE/ACM Transactions on Networking, vol. 18, no. 5, pp. 1610 - 1623, 2008.

[3] I. Rhee, A. Warrier, J. Min, L. Xu "DRAND: distributed randomized TDMA scheduling for wireless ad-hoc networks," IEEE Transactions on Mobile Computing, vol. 8, pp. 1384-1396, 2009.

[4] J. M. Holtzman, "Asymptotic analysis of proportional fair algorithm", In: $12^{\text {th }}$ IEEE International Symposium on Personal, Indoor and Mobile Radio Communications, 2001, vol. 2, pp. F33-F37.

[5] J. Zhang, F. Ren, and C. Lin, "Survey on transport control in data center networks", Network, IEEE vol. 27, no. 4, 2013.

[6] C. Pham, V. Lecuire and J.M. Moureaux "Performances of multihops image transmissions on IEEE 802.15.4 wireless sensor networks for surveillance applications", IEEE $9^{\text {th }}$ International Conference on Wireless and Mobile Computing, Networking and Communications (WiMob), 2013.

[7] E. Toscano, and L.L. Bello, "A novel approach for data forwarding in industrial wireless sensor networks," Emerging Technologies and Factory Automation (ETFA), pp. 1-10, 2010.

[8] M.R. Palattella, N. Accettura, L.A. Grieco, G. Boggia, M. Dohler and T. Engel, "On optimal scheduling in duty-cycled industrial IoT applications using IEEE802.15.4e TSCH", Sensors Journal, IEEE, vol. 13 , no. 10, pp. 3655-3666, 2013.

[9] M. Li, Y. Jing and C. Li "A robust and efficient cross-layer optimal design in wireless sensor networks", Wireless Personal Communications, vol. 72, no. 4, pp. 1889-1902, 2013.

[10] A.A. Rezaee, M. Hossein, A. Yaghmaee, and M. Rahmani, "Optimized congestion management protocol for healthcare wireless sensor networks", Wireless Personal Communications, vol. 75, no. 1, pp. 11-34, 2014.

[11] W. Wei, and Y. Qi, "Information potential fields navigation in wireless Ad-Hoc sensor networks" Sensors, vol. 11, no. 5, pp. 4794-4807, 2011.

[12] W. Wei, P. Shen, Y. Zhang, and L. Zhang, "Information fields navigation with piece-wise polynomial approximation for highperformance OFDM in WSNs", Mathematical Problems in Engineering, vol. 13, Article: 901509, 2013. 
[13] W. Wei, Q. Xu, L. Wang, X.H. Hei, P. Shen, W. Shi and L. Shan, "GI/Geom/1 queue based on communication model for Mesh Networks“, International Journal of Communication Systems, DOI: 10.1002/dac.2522, 2012.

[14] W. Wei, Y. Qiang, J. Zhang, "A bijection between lattice-valued filters and lattice-valued congruences in residuated lattices", $M a$ thematical Problems In Engineering, vol. 2013, Article: 908623, 2013.

[15] W. Wei, L. Zhang, D. Guo, and P. Shen, " Applications of information navigation method in wireless sensor networks", Journal on Communications, vol. 33, no. Z2, pp. 146-152, 2012.
[16] W. Wei, X. Yang, B. Zhou, and P. Shen, "Holes detection in anisotropic sensornets: topological methods", International Journal of Distributed Sensor Networks, vol. 2012, Article ID 135054, 2012

[17] W. Wei, X. Yang, B. Zhou, and J. Feng, "Combined energy minimization for image reconstruction from few views" , Mathematical Problems in Engineering, vol. 2012, Article ID 154630, 2012.

[18] W. Wei, H.M. Srivastava, Y. Zhang, L. Wang, P. Shen, and J. Zhang, "A local fractional integral inequality on fractal space analogous to anderson's inequality", Abstract and Applied Analysis, vol. 14, Ariticle ID: 797561, 2014.

Received: September 16, 2014

(C) Zhang et al.; Licensee Bentham Open.

This is an open access article licensed under the terms of the Creative Commons Attribution Non-Commercial License (http://creativecommons.org/licenses/by-nc/3.0/) which permits unrestricted, non-commercial use, distribution and reproduction in any medium, provided the work is properly cited. 